\title{
Addictive Links: The Motivational Value of Adaptive Link Annotation
}

Peter BRUSILOVSKY, Sergey SOSNOVSKY, and Michael YUDELSON

\begin{abstract}
Adaptive link annotation is a popular adaptive navigation support technology. Empirical studies of adaptive annotation in the educational context have demonstrated that it can help students to acquire knowledge faster, improve learning outcomes, reduce navigational overhead, and encourage non-sequential navigation. In this paper, we present our exploration of a less known effect of adaptive annotation, its ability to significantly increase student motivation to work with non-mandatory educational content. We explored this effect and confirmed its significance in the context of two different adaptive hypermedia systems. The paper presents and discusses the results of our work.

Keywords - Adaptive hypermedia, navigation support, visual cue, adaptive annotation, motivation, e-learning, empirical study, assessment.
\end{abstract}

\section{INTRODUCTION}

A

DAPTIVE link annotation is a powerful personalization technology in adaptive educational

hypermedia and e-Learning systems. The idea of adaptive annotation is to augment links with personalized hints that inform the user about the current state of nodes behind the annotated links. Usually, these annotations are provided in the form of visual cues employing, for example, contrasting font colors, sizes, and types for the link anchor or different icons next to the anchor (Brusilovsky, 2007). A range of annotation approaches has been explored in many Web-based educational hypermedia systems (Brusilovsky and Eklund, 1998; De Bra and Calvi, 1998; Henze and Nejdl, 2001; Papanikolaou et al., 2003; Weber and Brusilovsky, 2001). Empirical studies of adaptive annotation in the educational context have demonstrated that it can help students acquire knowledge faster (Brusilovsky and Pesin, 1998; Masthoff, 2002), improve learning outcomes (Davidovic et al., 2003; Specht, 1998), reduce navigational overhead (Brusilovsky and Eklund, 1998; Brusilovsky and Pesin, 1998; Masthoff, 2002), and encourage non-sequential navigation (Brusilovsky and Eklund, 1998). These effects are well known and are frequently cited as being the reason to use adaptive annotation.

Much less explored is the ability of adaptive annotation to significantly increase student motivation to work with non-mandatory educational content. This effect was first discovered during studies of the ELM-ART system (Weber and Specht, 1997). While the magnitude of 
the effect was large (the number of visited pages for a version with adaptive navigation support was twice as larger than for a version without it), it was not statistically significant due to a small sample size and the motivational value of adaptive annotation went unnoticed by the community. More recently, our team re-discovered the motivational value of adaptive annotation in a study of the QuizGuide adaptive hypermedia service (Brusilovsky et al., 2004). The presence of adaptive annotation in QuizGuide encouraged students to try twice as many questions, as well as to access a significantly larger diversity of questions. In some sense, adaptive annotations made the work with quizzes almost addictive: Once the students started a session, they stayed with the system significantly longer doing significantly more work. The average session length (measured in terms of questions attempted per session) nearly doubled (Brusilovsky and Sosnovsky, 2005).

Despite the significance of the results obtained in the QuizGuide study, a single study was not deemed sufficient to pronounce the motivational effect of adaptive annotation as being a prime reason to use this technology. Moreover, QuizGuide used a rather nontraditional topic-based adaptation mechanism. It was not evident whether the same effect could be observed with a classic concept-based adaptation mechanism, with different kinds of educational content, and in different domains. Over the last three years, we performed a larger scale exploration of the "addictive links" effect. We developed two adaptive hypermedia systems, which differ from the original QuizGuide in at least two of three aspects: type of adaptation mechanism, type of content, and learning domain. We also performed several classroom studies of these systems to explore the motivational and other values of adaptive link annotation in a realistic course-length context. Our studies confirmed our original observations in both explored systems, demonstrated the significance of the observed effect, and brought a better understanding of some underlying mechanisms. This paper presents an account of this work. We start with a brief review of early studies of the 
motivational effect in ELM-ART and QuizGuide (section II). After that, we introduce two newer systems, NavEx and SQL Guide, that were used to confirm the motivational effect (sections III and IV), and present the results of the classroom studies of these systems (sections V and VI). At the end, we discuss the importance of our findings in the context of solving the problem of increasing student motivation in E-Learning (section VII), and chart some directions for future research (section VIII).

\section{ELM-ART And QuizGuide - The Discovery of the Motivational EfFect}

ELM-ART (Weber and Brusilovsky, 2001), one of the first Web-based adaptive hypermedia systems, used concept-based adaptive link annotation to guide students to the most appropriate pages, examples, and problems in an interactive textbook for the LISP programming language. For example, a red bullet before a link to a page indicated that the student is not ready yet to learn the content of this page, while a green bullet indicated recommended content, i.e., interesting and ready to be learned (Fig. 1). The motivational effect of adaptive link annotation was discovered in an out-of-classroom study of ELM-ART. The system was freely available on the Web for anyone interested in learning LISP. The use of the system was not mandatory - the users worked with the system only as long as they were interested and motivated. The log analysis reported in (Weber and Specht, 1997) demonstrated that users who were familiar with at least one programming language and worked with adaptive link annotations visited almost twice as many pages (23.0 vs. 13.1 pages) and solved more exercises and problems then similar users working without annotations. The authors noted, however that the "effect is not quite statistically significant in our small sample" reporting $p=0.11$. In addition, there was no effect of adaptive link annotation for users who were absolute novices in programming, indicating that this effect may depend on user background knowledge level (Brusilovsky, 2003). 


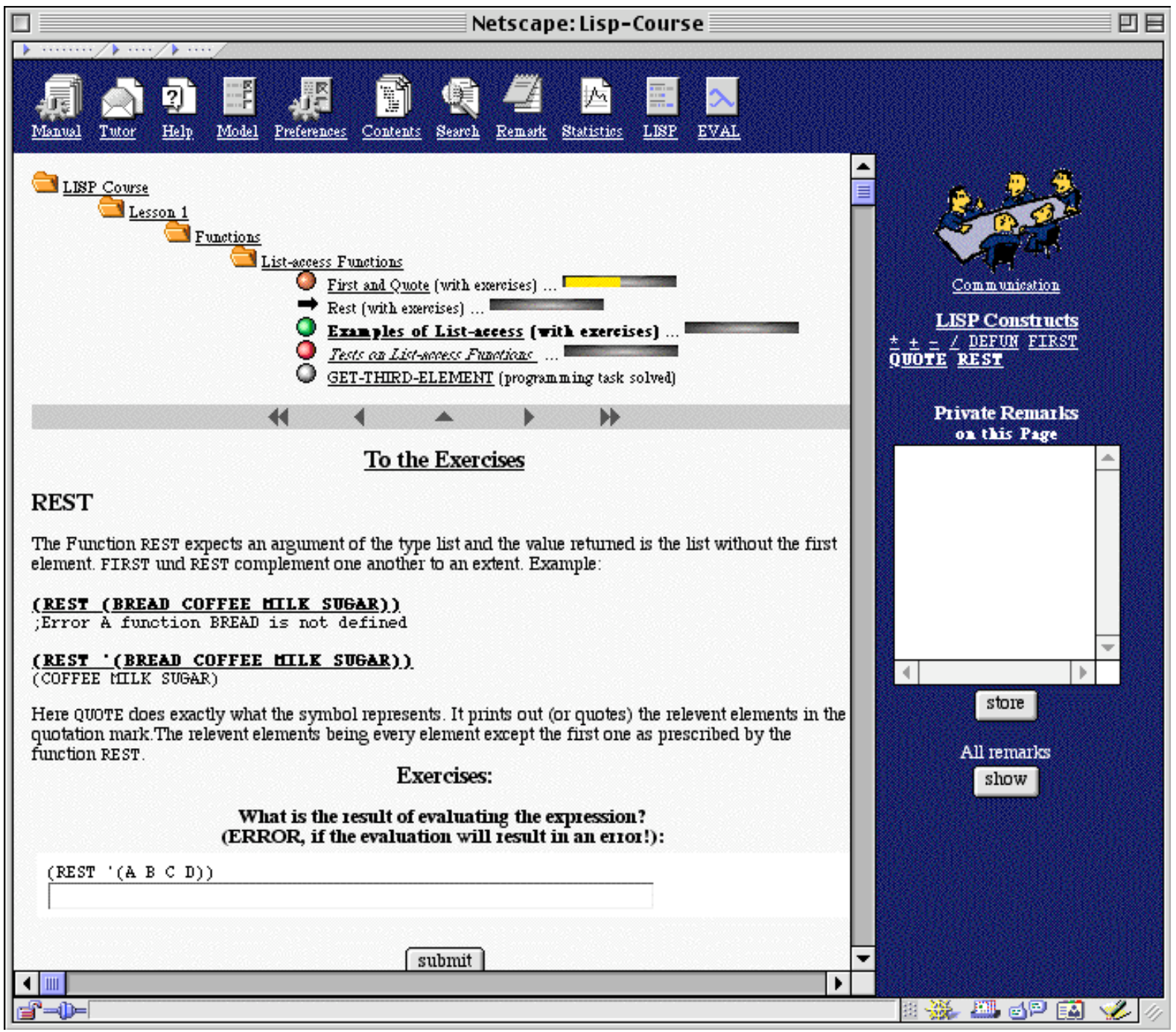

Fig. 1. Adaptive link annotation in ELM-ART: Red bullets indicate pages, which the system perceives the user is not ready for. Green bullets indicate recommended (ready to learn) pages.

QuizGuide (Brusilovsky et al., 2004), is an adaptive hypermedia service providing personalized access to self-assessment quizzes on $\mathrm{C}$ programming. It uses adaptive link annotation for the same purpose as ELM-ART and many other adaptive educational hypermedia systems - to guide students to the most appropriate learning content at a given time. However, the form of QuizGuide's annotation is relatively different: it focuses on the student's current knowledge and learning goals, as represented in terms of coarse-grained topics. Instead of indexing quizzes and questions with traditional fine-grained concepts, QuizGuide groups them into topics, which represent large chunks of domain knowledge (Fig. 2). The link to each topic is annotated with an icon showing a target with (or without) arrows. 
The number of arrows (from 0 to 3 ) reflects the student's performance on all quizzes that belong to the annotated topic (no arrows represent none or very little progress, while three arrows indicate a high level of understanding). The color of a target encodes the relevance of a topic to the current learning goal of the entire class. Current topics (the focus of the most recent lecture) are annotated with bright-blue targets. Topics that serve as prerequisites for any of the current topics are marked with pale-blue targets. Completed topics are assigned grey targets. Finally, topics that belong to the learning goals not yet covered in class are annotated with crossed targets. The original motivation of this design was to increase the quality of learning and the success rate of student's answers by guiding them to the most appropriate quizzes. Both are effects that adaptive link annotation is known to deliver in the educational context (Brusilovsky, 2007).

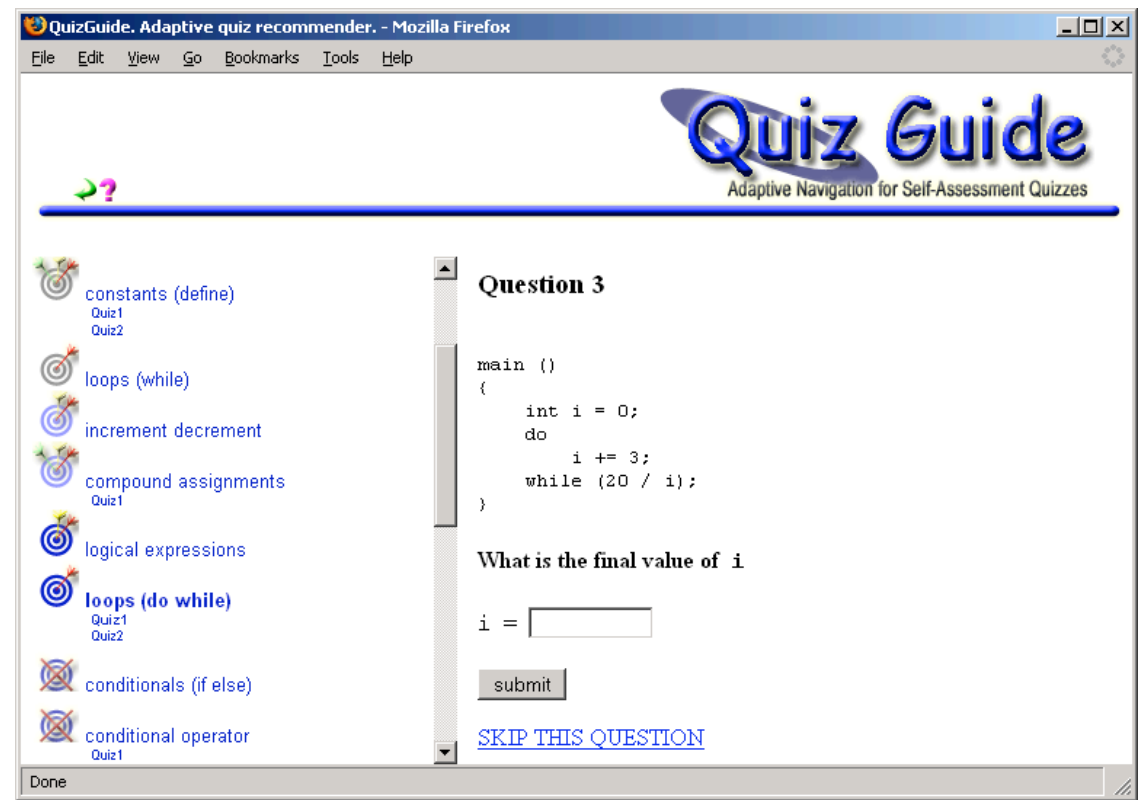

Fig. 2. The interface of QuizGuide

During the classroom study, QuizGuide was made available to students as one of the tools presented on the course portal. Within the course portal, the students were able to access the same set of self-assessment quizzes in either the traditional way (without annotation) as 
well as through QuizGuide (with adaptive annotation). The use of self-assessment quizzes was not mandatory - the students were allowed to use them as much as they wanted, in order to check the level of their knowledge and to prepare for classroom quizzes and exams. The value of adaptive link annotation was assessed by comparing the activity logs and the learning outcomes of the students between different classes and within a single class. While the analysis demonstrated that the original goal was achieved (in the presence of adaptive annotation the success rate of students' answers and the overall knowledge gain increased significantly) (Brusilovsky and Sosnovsky, 2005), the most striking effect of adaptive annotation was the remarkable increase of student motivation to work with self-assessment quizzes (Brusilovsky and Sosnovsky, 2005; Brusilovsky et al., 2006). As we mentioned above, with the added adaptive annotations, the system became remarkably more "addictive". The value of all non-capped performance parameters doubled or nearly doubled. For example, the average number of question attempts over the course duration increased from 128 to 261 . The average number of different quizzes attempted by a student within a single session increased from 1.87 to 3.64. All results were statistically significant. The values of most capped performance parameters also increased significantly, i.e., with QuizGuide, a significantly larger percentage of students was involved in active work with the system (Brusilovsky et al., 2006).

\section{NAvEX - AdAPtive Guidance For AnNotAted Code ExAMPles}

The NavEx system (Yudelson and Brusilovsky, 2005) provides adaptive access to a specific kind of educational content: interactive programming examples (about 70 examples are accessible through NavEx). NavEx is implemented as an adaptive, value-added service for a non-adaptive system, WebEx (Brusilovsky and Yudelson, 2008), which delivers the examples and supports student interaction with examples. NavEx groups all available examples into one learning tool and augments example links with adaptive visual cues. The 
NavEx window consists of a navigation frame (on left) and the content area (Fig. 3). Adaptive link annotations presented in the navigation frame integrate several kinds of information about examples and express them through the icon and font type.

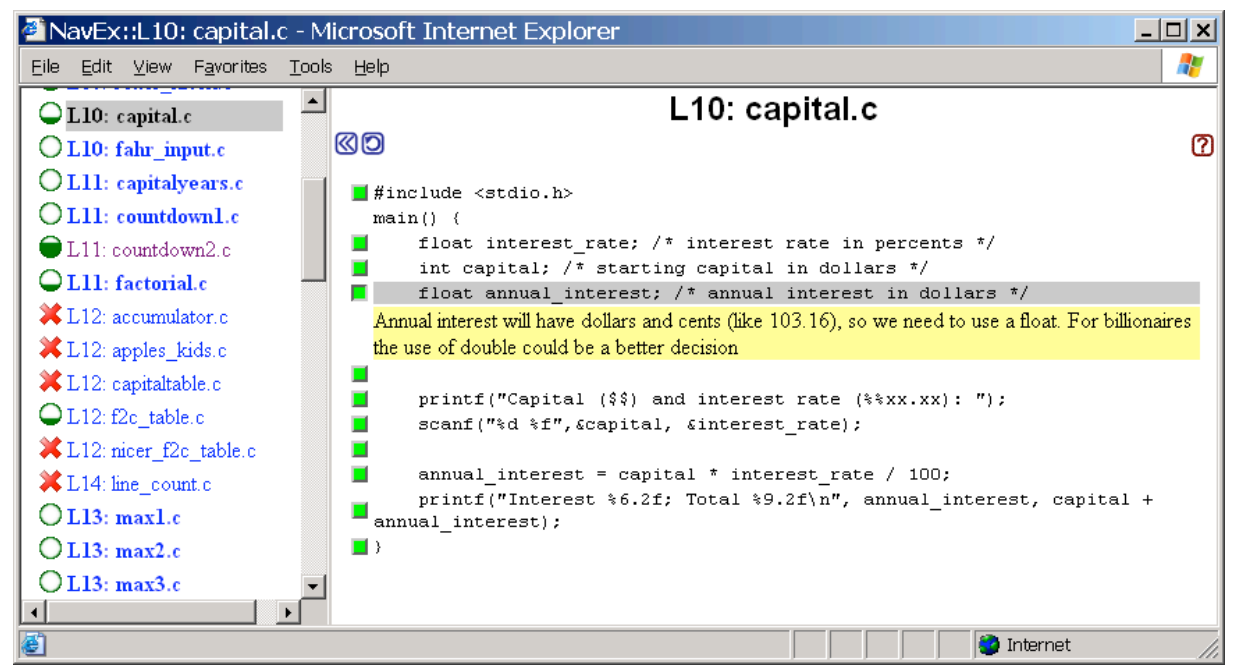

Fig. 3. The interface of NavEx

The icon/font combination displayed by NavEx attempts to combine two known approaches to adaptive link annotation. The annotation clearly indicates which examples are ready to be learned and which are not ready. This approach was pioneered on the Web in ELM-ART (Weber and Brusilovsky, 2001) and explored in a number of e-Learning systems such as AHA! (De Bra and Calvi, 1998) and KBS-HyperBook (Henze and Nejd1, 2001). In addition the annotation reflects the student's progress for each example. The progress-based annotation was pioneered on the Web in InterBook (Brusilovsky and Eklund, 1998) and further extended in INSPIRE (Papanikolaou et al., 2003) and other e-Learning systems.

In NavEx, examples that are not ready to be learned are marked with red-cross stop icons, similar to the red bullet in ELM-ART (Fig. 3). Ready to be learned examples are annotated with fillable circles. Depending on the amount of work already done by a student, within an example, the circle is empty, partially filled, or completely filled. NavEx distinguishes five progress levels from $0 \%$ to $100 \%$, with $25 \%$ increments (Fig. 3). An empty 
green bullet denotes examples that are available, but not yet explored by the student. To distinguish ready and recommended examples (an analogy to the green bullet in ELM-ART) from ready but not recommended (not interesting due to the current level of knowledge), links to recommended examples are displayed in bold font.

The decision which annotation icons are displayed is made by the concept-based navigation support mechanism (Brusilovsky and Yudelson, 2008), which takes into account the current level of student knowledge and the list of programming concepts associated with each example. An interesting innovation applied in NavEx is the fully-automatic indexing of examples with prerequisite and outcome concepts. First, a special parser generates the concept list for every example. Then, the resulting lists are separated into prerequisite and outcome concepts based on the course structure and the order of the examples. More details of this procedure can be found in (Sosnovsky et al., 2004).

Whenever a student opens comments for a code line within an example, WebEx sends a corresponding event to the user model. Once an example is sufficiently explored, all of the example concepts are considered known. The level of "sufficiency" is defined by the ratio of example's concepts that are already known to the total number of concepts in the example. A higher ratio means that fewer comments have to be viewed to sufficiently explore an example. The exact formulas for user modeling and icon selection can be found in (Yudelson and Brusilovsky, 2005).

The stop icon indicates that the student does not know some of the prerequisite concepts of an example. The more examples the student browses, the more concepts he or she learns, thus the smaller number of examples that are annotated with stop icons. Following the spirit of adaptive link annotation the stop icons warn but do not forbid students to access examples; they simply indicate that the links are not recommended navigational paths. The 
students are free to make their own decisions, even if it is against the system's recommendation.

It is worth mentioning here that the conceptual "knowledge" that NavEx deals with is not the same knowledge a student would get from problem solving. Navigating examples simply facilitates comprehension - only one of the tiers in Bloom's cognitive behavior taxonomy (Bloom, 1956). Although seemingly inferior to application or analysis levels, the comprehension still constitutes an important aspect of student's mastery of the domain.

\section{SQL-Guide: ConcePt-Based NAVigation Support for Database Programming PROBLEMS}

SQL-Guide is an adaptive hypermedia service offering students personalized access to interactive problems in the domain of SQL-programming. Each problem requires the student to write an SQL query for a given task and a set of sample databases. The student's answers are evaluated on-the-fly, and a limited amount of feedback is provided. An important feature of SQL-Guide is the dynamic problem generation. Every problem is, essentially a template capable of generating a random problem based on the set of pre-defined features. A single template is capable of producing from a dozen to several hundred unique problems. As a result, when a student returns to a problem accessed before, the problem definition, the original database and, consequently, the answer to the problem are different. This allows the students to master a certain skill through a sequence of typical exercises. Every template is associated with a coarse-grained topic and a set of concepts, thus providing the basis for evaluating the student's knowledge and adapting to it in two ways.

As an adaptive service, SQL-Guide resides between the individual user and a set of problems, trying to direct the user to the most appropriate problems. Following the QuizGuide model, SQL-Guide groups problems into coarse-grained topics and annotates topic links with adaptive "target-arrow" icons, where the number of arrows reflects the current level of knowledge of the topic, and the color of the target indicates the relevance of a 
topic to the current learning goal of the class (Fig. 4a). SQL-Guide attempts to expand this approach by complementing topic-based annotation with an additional layer of concept-based annotation icons provided for the links to individual problems within each topic (see Fig. 4b).

Every icon associated with a problem represents the cumulative level of knowledge the student has demonstrated for the set of concepts underlying this problem. In addition, problems answered correctly receive a checkmark icon. Thus, while SQL-Guide uses the same concept-based navigation mechanism as NavEx (based on prerequisite-outcome indexing of problems with domain concepts), the adaptive concept-based annotations generated by SQL-Guide emphasize the progress-oriented approach of the original QuizGuide, instead of the ready/not ready approach of ELM-ART. To investigate whether the concept-based progress-oriented annotations provide any additional value in the presence of topic-based ones, we have developed another version of SQL-Guide, which disables the concept-based adaptation layer (Fig 4a).

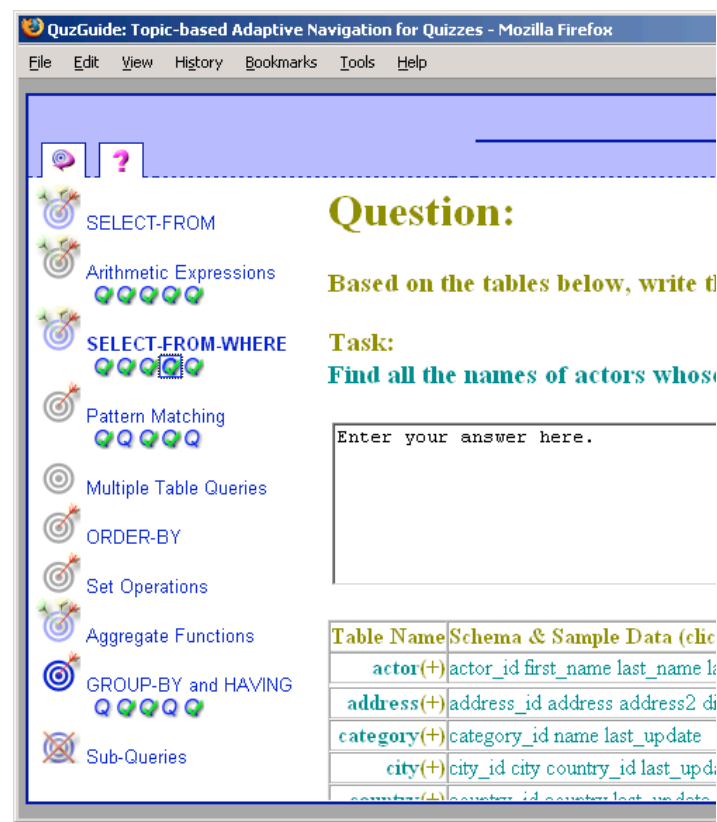

a)

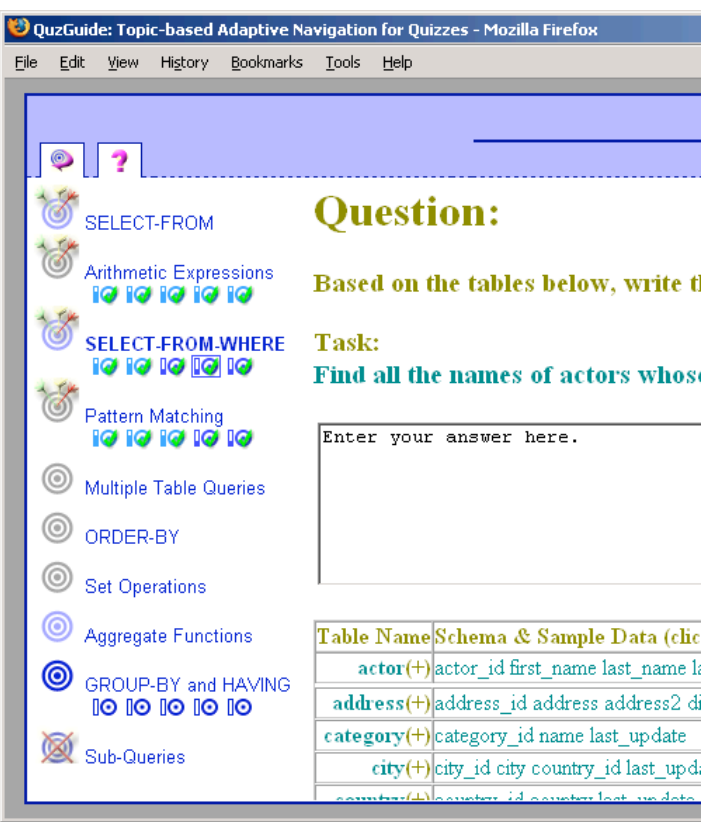

b)

Fig. 4. Adaptive navigation for SQL-KnoT problems provided by SQL-Guide with disabled (a) and enabled (b) layers of concept-based annotations. 
Fig. 5. summarizes all annotations used in both versions of SQL-Guide interface. Large "target-arrow" icons (Fig. 5a) annotating topics are used in both versions; however, on the problem level, the interfaces differ. The only navigation support provided by the pure topicbased SQL-Guide for individual problems is a checkmark denoting that a problem was solved correctly at least once (Fig. 5b). The interface combining topic-based and concept-based adaptation annotates problems using small targets with vertical progress bars (Fig. 6c). The student progress with the concepts underlying the problem is double-coded: as the knowledge level grows, the bar level rises and the icon fades. By means of this abstraction, SQL-Guide tries to deliver two kinds of information to the student: where the progress has been made (higher bar level) and where the attention should be focused (brighter target color). To help a student understand the meaning of annotations, SQL-Guide dynamically generates mouseover hints for all icons. Detailed help explaining all interface elements is available as well.

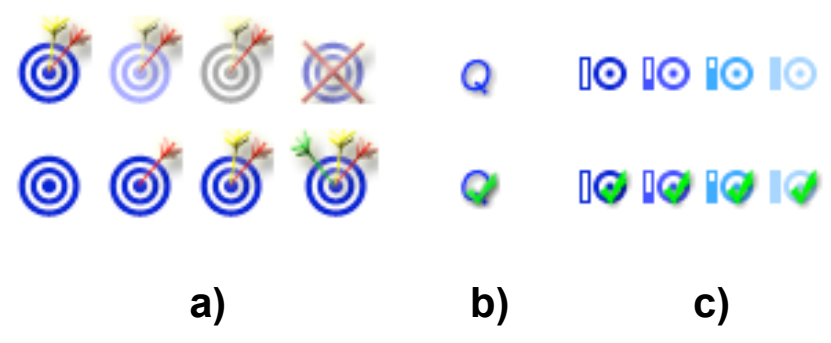

Fig. 5. SQL-Guide annotations: (a) Topic-based: upper row - levels of topical relevance to the current learning goal (current goal, prerequisite for the current goal, passed goal, future goal); lower row - knowledge levels for the topic (from 0 to 3); (b) Problem progress (done / not done); (c) Concept-based (four knowledge levels combined with problem progress bar).

\section{A Study of the Concept-Based Navigation Support in NavEx}

We performed a number of classroom studies for both the non-adaptive WebEx system, and the adaptive value-added service NavEx. The studies reported below were done in the context of an undergraduate programming course at the School of Information Sciences, University of Pittsburgh, from the Fall 2003 to the Fall 2004 semesters. The non-adaptive WebEx was accessible to students starting in the Fall 2003 semester. NavEx was introduced in the Fall 2004 semester. After students gained access to adaptive interface (NavEx), the 
non-adaptive access to examples (WebEx) was still available - through non-annotated links in the course portal. In addition to annotated examples available via WebEx and/or NavEx, the course portal had links to relevant reading materials as well as self-assessment quizzes. All of these tools were optional to use. The setup of the course remained the same across all semesters, preserving the instructor, lecture content, scope of home assignments (largely dealing with writing a small piece of code), and in-class quizzes. There were about 70 distinct annotated examples spread over 22 lectures made available to students, 60 of which actively participated in the studies across all 3 semesters.

The introduction of an adaptive service caused an impressive increase in student interaction with examples, compared to their work with non-adaptive interfaces in previous semesters. Due to the fact that the only change in the interface for accessing annotated examples was an extra frame with annotated links in NavEx and that the work with educational content continued to be non-mandatory, the obtained data were interpreted as another demonstration of the motivational effect of the adaptive link annotation. The following subsections present some results, demonstrating both the quantitative and qualitative effect of NavEx on the profile of student work done with annotated examples.

\section{A. Bottom-line Data and Significance}

In this section, we will present our quantitative analysis of the added value that the adaptive annotations in NavEx have over non-adaptive access through WebEx. The source data for the analysis were the activity logs collected by the systems. The logs recorded every user click (i.e., selecting an example to view or clicking on a line of example code). Data collection procedures did not differ across discussed semesters and were not dependent on the method students used to access code examples (whether via adaptive or non-adaptive systems). Student work with any of the discussed systems was included equally for user 
modeling. Log data gave clear evidence as to whether a student accessed quizzes or examples through the adaptive service or not.

We used three variables to parameterize student performance:

1. activity: the number of clicks on lines of code (in the case of WebEx and NavEx)-later referred to as clicks or actions;

2. quantity: the number of examples explored (WebEx and NavEx)-later referred to as examples, and

3. coverage: the number of lectures that the reviewed examples were drawn from (later referred to as lectures).

Each of these variables was aggregated on two levels:

4. overall performance level - the total number of clicks made, examples explored, and lectures covered by each user over the course of the semester; and

5. session performance level - the average number of clicks made, examples explored, and average number of lecture topics explored per session by a user (also referred to later as average session statistics).

Our objective was to determine whether activity, quantity and coverage of topics were higher for students who were exposed to NavEx than for those who used the non-adaptive WebEx format. We inspected nearly 3,400 WebEx and NavEx actions (requests for comments to the lines of code). Prior to the data examination, we performed outlier filtering with regards to the overall number of clicks made by a student over the semester. The filtering was done by setting a plus-minus three-sigma interval around the mean for the overall number of clicks. The distributions of data across all our variables were severely skewed because there were a number of not very active students (in terms of clicks made, examples reviewed, etc.), fewer were moderately active students, and very few were extremely active students. This, along with heterogeneity of variances, prevented us from 
applying parametric statistical tests to compare the usage data. Instead, we employed nonparametric Mann-Whitney tests as t-tests' substitutes. The results revealed that values of nearly all performance measures across all aggregation levels were significantly higher for users exposed to the adaptive features of NavEx (Table 1).

Table 1. Comparing the means of the variables for semesters when the adaptive system (NavEx) was used in combination with the non-adaptive one (WebEx) vs. semesters when only non-adaptive systems were used.

\begin{tabular}{|c|c|c|c|c|}
\hline & & WebEx & NavEx + WebEx & jalue \\
\hline & Clicks & $34.76 \pm 6.66$ & $171.90 \pm 65.56$ & $<0.001 * * *$ \\
\hline Overall user & Examples & $5.66 \pm 0.87$ & $18.10 \pm 4.32$ & $<0.001 * * *$ \\
\hline & Lectures & $3.52 \pm 0.42$ & $8.20 \pm 1.23$ & $<0.001 * * *$ \\
\hline Average & Clicks & $7.85 \pm 0.87$ & $9.49 \pm 1.28$ & 0.122 \\
\hline user session & Examples & $1.56 \pm 0.12$ & $2.03 \pm 0.22$ & $0.013 *$ \\
\hline statistics & Lectures & $1.20 \pm 0.05$ & $1.37 \pm 0.10$ & $0.020 *$ \\
\hline
\end{tabular}

* significant at the 0.05 level, *** significant at the .001 level

On the level of overall statistics, users exposed to adaptive guidance were making 5 times more clicks a semester (an average of roughly 170 vs. 35), reviewing almost three times as many code examples (an average of roughly 18 vs. 6), and covering almost twice as many lectures (an average of 8 lectures vs. 3.5). All of the mentioned differences were significant. On the level of average user session statistics, the number of examples reviewed and number of lectures they were drawn from were significantly higher for users of adaptive guidance.

To ensure that the observed difference in user activity was not explained by differences in student population across semesters, but was rather an indicator of the added value of adaptive annotation, we analyzed the students' initial programming experience and gender across semesters. The results of the Chi-Square test show that there was no significant 
difference between the WebEx and NavEx groups in gender distribution (Chi-Square $=1.720$, $\mathrm{p}$-value $=0.268)$ or initial experience distribution $($ Chi-Square $=0.704, \mathrm{p}$-value $=0.703)$.

We could not distill the effect of browsing the annotated examples on the student performance in-class (quizzes, midterm exam, final exam) during the reported studies. This can be attributed to the fact that WebEx/NavEx were only a part of a fleet of tools including self-assessment quizzes, which are known to significantly impact performance on their own. However, the studies where WebEx was used alone, without "competing" online educational tools (Goreva et al., 2007; Yudelson et al., 2008) demonstrated significant positive effect of work with annotated examples on code understanding (that WebEx promotes).

\section{B. Navigational Pattern Analysis}

While comparing student work with adaptive and non-adaptive versions, we noticed that typical NavEx sessions are both longer and more diverse than WebEx-only sessions are: Students explored a larger number of examples through NavEx. In addition, students more frequently accessed activities corresponding to different lectures within the same session.

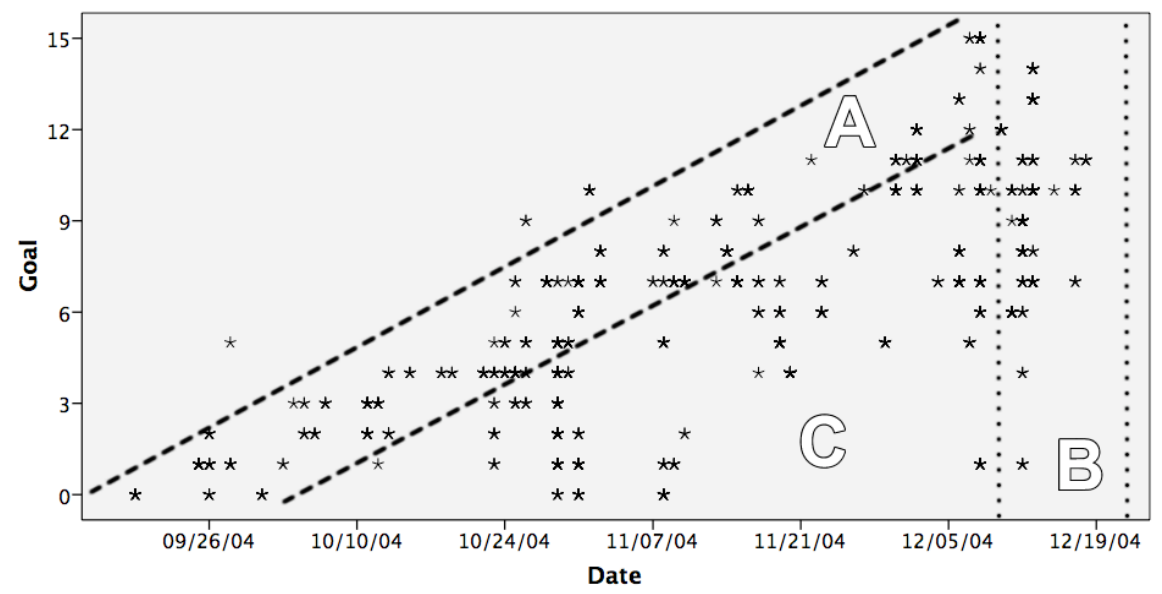

Fig. 6. Time distribution of all actions performed by students with NavEx and WebEx in the Fall 2004 semester. Zone "A"lecture stream, zone "B" - final exam cut, and zone "C" - self-motivated work with materials from earlier lectures.

To take a closer look at the nature of these results, we performed a deeper analysis of student activity, by taking into account lecture coverage included in all students' actions. 
Every selection of an example of a question was attributed to the lecture (or the learning goal) it belonged to. For example, Fig. 6 visualizes over 1,500 clicks on examples performed by students using NavEx of WebEx in the Fall 2004 semester. Fifteen lectures form the vertical axis. The time of the action is marked on the horizontal axis. We can detect three zones of activity. Zone "A" contains the "current" activity that students perform along the lecture stream of the course. It is fairly broad, since homework assignments (offered every other week consistently throughout reported semesters) introduce a 1-2 week delay in shifting the students' focus from the previous topics. Zone "B" contains a period of preparation for the final exam. The pattern of work with our systems is totally different during this time. Finally, zone "C" contains all actions that students performed during the regular part of the semester, for topics located far from the current lectures, possibly in an attempt to bridge a gap in their knowledge that should have been acquired earlier. This is the zone we were particularly interested in: we wanted to check whether prerequisite-based adaptive annotations were effective in helping NavEx users to reinforce their knowledge by coming back to the examples from earlier lectures.

To find that out we used two measures to assess the intensity of students' selfmotivated activity: the number of actions in zone "C" divided by the total number of actions and the average distance between the learning goal which is current at this time and their current activity. For the calculation of the second measure, we used zones "A" and "C". For all students we calculated an average "C"-ratio and goal distance for examples. To evaluate the influence of NavEx on motivated activity, we again divided students into two groups: adaptive (Fall 2004) and non-adaptive (Spring 2004 and Fall 2003). Since assumptions of homogeneity of variance and normality were violated, we had to perform nonparametric Mann-Whitney's test. As shown by Table 2, both measures are significantly higher for the adaptive group than for non-adaptive one. 
Table 2. These parameters characterize the self-motivated activity of students with and without adaptive annotations: the "C"-ratio estimates the percentage of students' activity performed outside the current course focus, while the Goal distance assesses how broadly (in terms of learning goals) the voluntary interest of a student roams when working with the system.

\begin{tabular}{cccc}
\hline & $\begin{array}{c}\text { Non-adaptive } \\
(\text { WebEx })\end{array}$ & $\begin{array}{c}\text { Adaptive } \\
\text { (NavEx) }\end{array}$ & p-value \\
\hline "C" ratio & $0.24 \pm 0.05$ & $0.51 \pm 0.08$ & $0.005^{* *}$ \\
Goal distance & $8.73 \pm 1.90$ & $17.64 \pm 2.51$ & $0.002^{* *}$ \\
\hline & $* *$ significant at the .01 level
\end{tabular}

The observed data provide evidence that progress-based and prerequisite-based adaptive annotations generated by NavEx increased the amount of student work not only quantitatively, but also qualitatively. In NavEx, the zone of recommended work is adapted to the student's individual progress. It provides a stronger push to explore insufficiently learned prerequisite concepts in previous examples and changed the distribution of their work along the course directing students' attention to the material related to earlier lectures which they may not understand well.

\section{EXPLORING THE VALUE OF COMBINED AdAPTIVE NAVIGATION SUPPORT IN SQL-Guide}

We evaluated SQL-Guide in two classroom studies in the Fall 2007 semester. The system was used as a learning tool in one graduate and one undergraduate database class (38 and 36 students, respectively) taught by the same instructor. Each version of the system provided access to 46 problem templates. Each template was accessible in two waysthrough SQL-Guide (the adaptive mode) and through the learning portal (non-adaptive mode). To access SQL-Guide students needed to login into the learning portal as well. Within SQL-Guide, templates were grouped into 10 topics (Fig. 4).

Within the course portal, they were placed in the corresponding lecture folder. Both adaptive and non-adaptive versions of SQL-problems were equally accessible through the course; no additional incentive to use the adaptive mode was introduced. All user interactions 
with the systems were logged. For every problem-solving attempt, the associated log record contained template ID, time of the attempt, access point (SQL-Guide or portal), and the attempt result (success or failure). In total, 19 graduate students and 26 undergraduate students attempted at least one problem during the semester.

\section{A. The Motivational Effect: Confirming the Value of Adaptive Annotation}

The first goal of this classroom study was to check whether the motivational effect of topic-based navigation support discovered in QuizGuide (a system for the C-programming domain) could be transferred to a different domain (SQL programming) with a different kind of learning activity (C-questions emphasized program understanding, while SQL-problems emphasized program generation). To assess the value of adaptive navigation support in SQLGuide, we looked at such usage parameters as the number of attempts, the number of distinct problems attempted, the number of topics for which at least one problem had been attempted, the number of sessions and the average session length. Overall, students accessed 4,081 problems through SQL-Guide and 1,218 problems through the non-adaptive course portal. Because the system was introduced to the graduate students later in the course, the total number of attempts made by the undergraduate students was larger. Nevertheless, the observed difference was stable for both courses: adaptive access to the problems dominated non-adaptive access.

The results of the evaluation confirmed that the students were much more willing to access problems in the adaptive mode (through SQL-Guide). The magnitude of the effect was comparable to the earlier study of QuizGuide (Brusilovsky and Sosnovsky, 2005). On average, students from both courses made about three times more attempts in the adaptive mode than they did in the non-adaptive. They also accessed twice as many distinct problems and explored almost twice as many topics while receiving adaptive navigation support from SQL-Guide. The difference in the amount of work might have been caused by more frequent 
access (a greater number of sessions) and/or by longer sessions. For both these parameters we observed significantly higher values in the adaptive mode than in the non-adaptive mode. Thus, in agreement with our previous findings (Brusilovsky and Sosnovsky, 2005), in the presence of adaptive navigation support students not only accessed the system more often, but also stayed with the system longer and did more work per session: i.e., the use of the system becomes "addictive." Table 3 summarizes the results of statistical tests comparing major usage characteristics between the adaptive and non-adaptive modes. The Wilcoxon Matched-Pairs Signed-Rank Test was used in the analysis, since the parametric statistics assumptions were violated.

Table 3. Comparison of cumulative usage parameters for adaptive and non-adaptive access to SQL problems

\begin{tabular}{lccc}
\hline & Adaptive & Non-Adaptive & p-value \\
\hline Number of Attempts & 90.69 & 27.07 & 0.005 \\
Number of Attempted Problems & 23.11 & 10.80 & 0.019 \\
Number of Attempted Topics & 5.56 & 3.31 & 0.016 \\
Number of Sessions & 3.27 & 2.09 & 0.047 \\
Average Session Length & 21.99 & 10.62 & 0.021 \\
\hline
\end{tabular}

B. Determining the Added Value of Concept-based Adaptation

To investigate the difference between the two versions of SQL-Guide interface and determine the added value of the concept-based navigation, we divided students from both courses into two groups. Over the semester, the experimental group used the combined version of the system (Fig. 4b) while the control group had access to the pure topic-based interface (Fig. 4a). The non-adaptive access to the same set of SQL-KnoT problems, as well as all other course tools, was equally available for both groups. The groups were balanced with respect to the gender and pre-test scores. After filtering out outliers, the experimental group contained 13 students ( 7 undergraduate and 6 graduate), and the control group consisted of 15 students ( 8 undergraduate and 7 graduate). 
The analysis of main usage parameters (such as the number of attempts, the number of sessions, and the session length) did not reveal significant differences between groups. However, the non-parametric Mann-Whitney test indicated that students using the conceptbased interface, on average, made significantly more attempts per problem $(M=3.36$, $S D=2.66)$ than those using the topic-based interface $(M=1.68, S D=0.42)$, Mann-Whitney $U$ statistics $=51.0, p=0.033$ (we used the non-parametric Mann-Whitney test because the assumptions of parametric statistics did not hold).

Such an increase in problem persistence led to another statistically significant effect. The comparison of student knowledge levels taken from their user models at the end of the semester showed that the students from the concept-based group achieved higher knowledge levels $(M=0.45, S D=0.09)$ than the students from the topic-based group $(M=0.39, S D=0.03)$, $t(26)=2.71, p=0.023$

The next section analyzes the low-level navigational patterns followed by the students in SQL-Guide and reports on important differences in the distribution of these patterns between the experimental and the control group.

\section{Navigational Pattern Analysis}

To obtain a deeper understanding of how students of two groups work with SQL-Guide and respond to adaptive guidance, we performed a navigation pattern analysis of student sessions. The analysis revealed eight basic patterns of navigation: four problem-based patterns characterizing the transition of a student from one problem to another, three topicbased patterns reflecting the moves between topics and one combined pattern.

The problem-based patterns define students' navigational behavior within a topic. Transitions between problems in such situations are largely determined by the adaptive guidance provided on the level of problem icons and the feedback generated by SQL-Knot. The problem-based patterns can be subcategorized into the following: 
- Sequential: a student moves from one problem to another in the order they are placed inside the topic;

- Repetition: a student attempts the same problem again immediately after the previous attempt;

- Go-Back: a student decides to return to one of the previous problems in the same topic;

- Skipping: a student skips one or several problems by moving to the next problem within the same topic.

The topic-based patterns can be explained by the reaction of a student to the adaptive topic icons. Whenever a student decides to switch a topic, s/he can observe the current state of topic-based annotations and use it as a hint for choosing the most appropriate set of problems to work on. To differentiate the topic-based navigational decisions from the problem-based ones we identified the following three patterns:

- Next-Topic: a student moves to the first problem of the topic next in the list;

- Jump-Forward: a student moves to the first problem of a topic, which is more than 1 step further in the list;

- Jump-Backward: a student moves to the first problem of a topic earlier in the list.

Sometimes, when moving to a different topic, a student might decide to skip the rest of the problems of the previous topic and/or start the new topic, but not from the first problem. In these situations the student's behavior is influenced by both the topic icons on the top level and the problem icons within a topic. We considered such cases as combined patterns consisting of the corresponding topic-based pattern and one or two problem-based Skipping patterns (two Skipping patterns are registered if a student skips both the end of the previous topic and the beginning of the new one).

Fig 7. represents all the patterns in a graphical format. The distribution of the patterns is given in Table 4. 


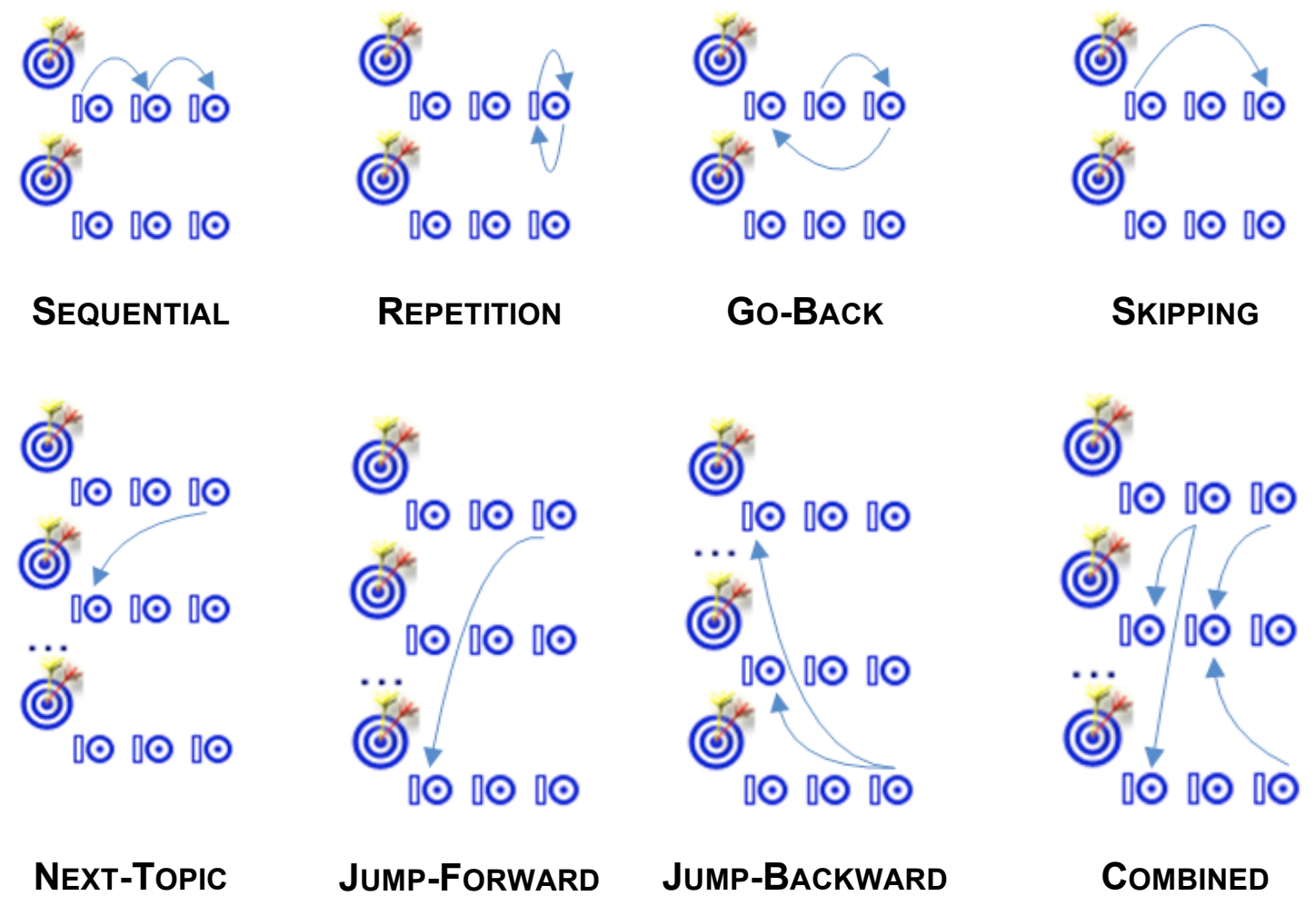

Fig. 7. Observed navigational patterns: the upper row shows problem-based patterns; while the lower row shows the three topic-based patterns and one combined pattern.

\begin{tabular}{l|l|r|r|r|r}
\hline \multirow{2}{*}{\multicolumn{2}{c|}{ Patterns }} & \multicolumn{2}{c|}{$\begin{array}{c}\text { With concept-based } \\
\text { navigation }\end{array}$} & \multicolumn{2}{c}{$\begin{array}{c}\text { Without concept-based } \\
\text { navigation }\end{array}$} \\
\cline { 3 - 6 } & \multicolumn{2}{c}{ Undergraduate } & Graduate & Undergraduate & Graduate \\
\hline \multirow{2}{*}{$\begin{array}{l}\text { Topic-based } \\
\text { patterns }\end{array}$} & Sequence & $22.54 \%$ & $44.44 \%$ & $43.77 \%$ & $52.37 \%$ \\
\cline { 2 - 6 } & Go-Back & $2.80 \%$ & $2.87 \%$ & $4.58 \%$ & $2.23 \%$ \\
\cline { 2 - 6 } & Skipping & $15.59 \%$ & $8.24 \%$ & $19.85 \%$ & $6.41 \%$ \\
\hline \multirow{3}{*}{$\begin{array}{l}\text { Problem- } \\
\text { based } \\
\text { patterns }\end{array}$} & Repetition-0 & $41.57 \%$ & $26.52 \%$ & $30.03 \%$ & $36.49 \%$ \\
\cline { 2 - 6 } & Repetition-1 & $17.51 \%$ & $17.92 \%$ & $1.78 \%$ & $2.51 \%$ \\
\cline { 2 - 6 } & Next-Topic & $42.05 \%$ & $73.68 \%$ & $37.14 \%$ & $70.91 \%$ \\
\cline { 2 - 6 } & Jump-Fwrd & $30.11 \%$ & $15.79 \%$ & $35.71 \%$ & $16.36 \%$ \\
\cline { 2 - 6 } & Jump-Bkwd & $27.84 \%$ & $10.53 \%$ & $27.14 \%$ & $12.73 \%$ \\
\hline
\end{tabular}

One of the goals of adaptive navigation support is to promote non-sequential navigation and guide students to relevant content in the most effective way. Analysis of the topic-based 
pattern distribution showed that on the level of topics, both versions of SQL-Guide encouraged students to choose topics in a non-linear order: the combined ratio of nonsequential patterns (Jump-Forward and Jump-Backward) varies from $65-70 \%$ for undergraduate students to $30-35 \%$ for graduate students.

The evaluation of SQL-Guide problem-based patterns showed that most of them are dominated by sequential problem access. The ratios of such patterns as Go-Back, Skipping did not exceed $20 \%$ for any of the group. The presence of the concept-based adaptation did not have any significant effect on the occurrence of these patterns. However, for the second most popular pattern - Repetition - the presence of the concept-based annotations had a dramatic effect. Generally, Repetition has two major sources depending on the result of the user's previous attempt:

- Repetition 0 : when the previous answer is incorrect, a student is likely to retake the same problem to remedy the error, try a different answer and, finally, get a checkmark for the problem;

- Repetition : $_{1}$ when the previous answer is correct, but the student still decides to solve the problem again.

While the Repetition $_{0}$ portion is stable across all the groups, introduction of conceptbased annotations increased the overall ratio of Repetition ${ }_{1}$ from $1.78 \%$ to $17.51 \%$ for the undergraduate students and from $2.51 \%$ to $17.92 \%$ in the graduate course. Hence, the adaptive concept-based problem icons caused students to repeat the same problem pattern not only when they failed it, but also when the problem had been solved correctly. The presence of a checkmark in both versions of the system ensured that the students were aware of the problem status, i.e., the students were consciously asking system to generate another problem with the same pattern after solving one correctly. Why did problem-level adaptive icons cause this change of behavior? It could be that these icons helped the students to realize that 
their knowledge of the concepts related to the problem is not strong (even after solving one instance of this problem) causing them to practice another instance right away. It could be that the students simply wanted to achieve a "higher score" indicated by the progress bars. We hope that future studies could reveal the source of the observed effect. In either case, this change of student behavior helps to explain two significant effects reported in the previous section:

- the average number of attempts per problem was greater for the experimental group, because students repeated the same problem more often in the presence of conceptbased adaptive navigational support on the problem level;

- the resulting knowledge levels were greater for the experimental group, because students worked more with each problem pattern either consciously trying to achieve a higher level of knowledge or simply trying to receive an icon with a higher level.

These results confirmed the added value of adaptive annotation on the concept-based level. The students who were able to observe adaptive icons, became more "addicted" to work not only globally, but on the level of a single problem pattern as well.

The observed effect may be considered from two sides. From one point of view, adaptive navigational cues caused students to become involved and motivated by the system, to work more and achieve better results. On the other hand, repeating the same problem pattern multiple times after one instance of a problem is solved correctly could be less optimal than switching to another problem, which could help students to practice not yet mastered concepts in a more different context. While repetitive work with the same problem pattern positively impacted student knowledge, we may speculate that a similar investment of time to work with a different pattern may have even larger positive impact. We may also speculate that while existing concept level guidance significantly and positively affected student work, this guidance was too simple to affect student behavior in the most positive 
way. Indeed, the first version of concept-level guidance included simple progress-based guidance, but lacked traditional three-state guidance (too simple, ready, too hard). Thus, our results not only show the effect of the concept-level adaptive guidance, but also suggest some ways to further improve it.

\section{DISCUSSION}

Motivation is a critical factor for successful learning. Higher motivation leads to better learning outcomes not only because of the increased amount of learning activity. It also improves cognitive engagement leading to better knowledge retention. The instructional strategies increasing motivation become overly important in the Web-based settings, as there is no teacher to provide reinforcement (Clark, 2002).

A number of approaches were suggested to improve student motivation in the context of Web-based learning (Hurley, 2008). For example, the learning material served by the system can be organized so that it provides the intrinsic motivation for students by implementing certain pedagogical and psychological strategies for fostering attention, confidence and relevance (Keller, 1987). Another promising approach is to model the level of motivation and rely on particular adaptive strategies directly addressing the problem of low motivation (Hurley and Weibelzahl, 2007).

Our studies demonstrate that adaptive link annotation presents a powerful addition to the list of already explored approaches. In the context of modern e-Learning, especially important is the ability of adaptive annotations to increase student's motivation to work with non-mandatory educational content. Over the last decade, researchers and practitioners have developed a range of advanced Web-based educational tools such as educational animations and simulations, on-line labs, tutorials, and self-assessment questions. Many of these developed tools have been evaluated in the lab and small-scale classroom studies and proven to be useful. However, we have now learned that the mere availability of a good tool, 
although known as beneficial for students, is not enough to ensure its broad educational impact (Naps et al., 2003). An important issue for those who research the use of computers in education is to increase the effective use of student-driven educational tools. Student-driven tools are created to assist student learning, yet their use is not required and does not count towards the student's course grade. Unlike a variety of assessment-driven tools that the students are required to use in order to complete their assignments, it is up to the students to decide to what degree and how frequently they use the student-driven tools. An instructor might work hard to provide a good set of educational tools of known benefit to the students, only to discover that these tools are really underused. Our work has demonstrated that adaptive annotation can be instrumental in motivating students to do more work with nonmandatory educational tools which is likely, in turn, to lead to better learning outcomes.

We have attempted to provide some insights into the mechanisms underlying the motivational value of adaptive annotation by analyzing the time-based distribution of students' activity (NavEx) and their transition patterns (SQL-Guide). Both analyses demonstrated that adaptive annotation seems to foster diversity in student navigation. When the examples are adaptively served by NavEx, the students have accessed significantly more examples that are farther from the current learning focus, than through the learning portal (non-adaptive). This is demonstrated by the fact that about one half of the topics-based transition patterns registered in SQL-Guide were non-sequential patterns (jump-forward and jump-backward).

At the same time the exploration of the added value of concept-based annotation in SQLGuide showed that the problem-based adaptive icons increased repetitive use of the same problem pattern, which may be less optimal than using other patterns related to the same concepts. Students became clearly influenced by problem-level guidance. In this case, a 
relatively weak progress-based guidance may not guide the students in the most optimal way. There are several possible remedies for this problem:

- "Rolling back": reducing the adaptive navigation support back to the topic-based level (in this case the students are less "addicted" to working with the same problem pattern and will be more frequently choosing different problem patterns within a topic);

- "Advancing forward": developing more accurate concept-based adaptive annotations guiding the students within a topic in a more optimal way;

- “Combining": integrating glass box (Höök et al., 1996) progress-based approach implemented currently in QuizGuide with direct guidance, a different kind of navigation support (this would allow the student to break less optimal patterns).

We plan to further investigate this issue and compare several possible strategies. One of the final goals of this study is the interface design for an e-Learning hypermedia service inheriting the addictive nature of adaptive navigation support and maximally promoting nonsequential navigation within the limits of in-class usage.

\section{CONCLUSIONS AND FUtURE WORK}

The results of our studies, reported in this paper, confirm the motivational effect of adaptive link annotation in educational hypermedia, demonstrate its magnitude and significance, and shed some light on the mechanisms of this effect. We were able to demonstrate this effect in the context of two different personalized access systems. The presence of adaptive annotations caused the increase of several usage parameters. When accessing non-mandatory educational activities through adaptively annotated links, the students explored significantly more activities, worked with them more persistently, and accessed items that had a broader distribution over course lecture topics. 
Added to the earlier report of a similar effect of adaptive annotation in the context of the ELM-ART study, our results allowed us to generalize the observations and talk about the motivational value of adaptive link annotation. We consider the results we obtained as both exciting and important. First, it is always exciting to discover new value in a popular technology. Secondly, the ability to significantly increase student motivation to interact more with non-mandatory educational activities turns adaptive annotation into a technology that may become critical to the practical success of a wide range of beneficial educational technologies.

More work is required to determine the borders of the motivational effect and to master its practical use. While this effect was observed with three different kinds of adaptive annotations, the mechanisms were conceptually similar, in that they combined appropriateness (too early, too late, just right) and progress-based (how much is already done) annotations. While we argue that both mechanisms contributed to the motivational value, we do not have data to confirm it. It is also not clear whether the observed effect is specific to these two annotation mechanisms or can be generalized to other kinds of adaptive annotations (and possibly to other kinds of adaptive navigation support). To answer these questions, we intend to continue our exploration of the motivational value of adaptive link annotation.

\section{ACKNOWLEDGEMENT}

This material is based upon work supported by the National Science Foundation under Grants No. 0310576, 042602, and 0447083. 


\section{REFERENCES}

B.S. Bloom, 1956. TAXonomy of EdUCATIONAL OBJECTIVES, HANDBOoK I: THE COGNITIVE Domain, New York: David McKay Co InC.

P. BRUSILOVSKY, 2003. ADAPTIVE NAVIGATION SUPPORT IN EDUCATIONAL HYPERMEDIA: THE ROLE OF STUDENT KNOWLEDGE LEVEL AND THE CASE FOR META-ADAPTATION. BRITISH JOURNAL OF EDUCATIONAL TECHNOLOGY, 34 (4), 487-497.

P. BRUSilovsky, 2007. AdAPTIVE NAVIGATION SUPPORT. IN: P. BRUSILOVSKy, A. KobSA AND W. NEIDL, EDS. ADAPTIVE NAVIGATION SUPPORT. LECTURE NOTES IN COMPUTER SCIENCE 4321. BERLIN HEIDELBERG NEW YoRK: SPRINGER-VERLAG,263-290.

P. BRUSILOVSKY AND J. EKLUND, 1998. A STUDY OF USER-MODEL BASED LINK ANNOTATION IN EDUCATIONAL hYPERMEDia. JOURNAL OF UNIVERSAL COMPUTER SCIENCE, 4 (4), 429-448.

P. BRUSILOVSKY AND L. PESIN, 1998. AdAPTIVE NAVIGATION SUPPORT IN EDUCATIONAL HYPERMEDIA: AN EVALUATION OF THE ISIS-TUTOR. JOURNAL OF COMPUTING AND INFORMATION TECHNOLOGY, 6 (1), 27-38.

P. BRUSILOVSKY AND S. SOSNOVSKY, 2005. ENGAGING STUDENTS TO WORK WITH SELFASSESSMENT QUESTIONS: A STUDY OF TWO APPROACHES. 10TH ANNUAL CONFERENCE ON INNOVATION AND TECHNOLOGY IN COMPUTER SCIENCE EDUCATION, ITICSE'2005 ACM PRESS, Monte de Caparica, Portugal, 251-255.

P. Brusilovsky, S. Sosnovsky and O. SHCHERbinina, 2004. QuizGuide: InCREASING THE EduCATIONAL VALUE OF INDIVIDUALIZED SELF-AsSESSMENT QUIZZES WITH ADAPTIVE NAVigation SUPPORT. IN: J. NALl AND R. ROBSON, EDS., WORLD CONFERENCE ON ELEARNING, E-LEARN 2004 AACE, WASHINGTON, DC, USA, 1806-1813.

P. BRusilovsky, S. Sosnovsky AND M. Yudelson, 2006. AdDICTIVE LINKS: THE MOTIVATIONAL VALUE OF ADAPTIVE LINK ANNOTATION IN EDUCATIONAL HYPERMEDIA. IN: V. Wade, H. Ashman AND B. SMYTh, EDS., 4TH INTERNATIONAL CONFERENCE ON ADAPTIVE HYPERMEDIA AND ADAPTIVE WEB-BASED SYSTEMS (AH'2006) LECTURE NOTES IN COMPUTER SCIENCE 4018, SPRINGER VerLag, Dublin, IRELAND, 51-60.

P. Brusilovsky and M. Yudelson, 2008. From WebEx to NavEx: Interactive ACCess to Annotated Program EXAmples. ProceEdingS of tHE IEEE, 96 (6), 990-999.

D. Clark, 2002. Psychological Myths In E-LEARning. MEDiCAL TEACHER, 24 (6), 598-604

A. DAVIDOVIC, J. WARREN AND E. TRICHINA, 2003. LEARNING BENEFITS OF STRUCTURAL EXAMPLE-BASED ADAPTIVE TUTORING SYSTEMS. IEEE TRANSACTIONS ON EDUCATION, 46 (2), 241-251.

P. De Bra and L. CAlvi, 1998. AHA! An open Adaptive Hypermedia ArChitecture. The NEW REVIEW OF HYPERMEDIA AND MULTIMEDIA, 4, 115-139. 
N. Goreva, M. Yudelson and B. Marshall, 2007. Using WebEx In A WeB APPLiCATion PROGRAMMING COURSE. ISSUES IN INFORMATION SYSTEMS, VIII (1-2), 52--57.

N. HENZE AND W. NEJDL, 2001. AdAPTATION IN OPEN CORPUS HYPERMEDia. INTERNATIONAL JOURNAL OF ARTIFICIAL INTELLIGENCE IN EDUCATION, 12 (4), 325-350.

K. HöÖK, J. Karlgren, A. WÆrn, N. DAhlbäck, C.G. Jansson, K. Karlgren AND B. LEMAIRE, 1996. A GLASS BOX APPROACH TO ADAPTIVE HYPERMEDIA. USER MODELING AND USER-ADAPTED INTERACTION, 6 (2-3), 157-184.

T. HURLEY, INTERVENTION STRATEGIES TO INCREASE MOTIVATION IN ADAPTIVE ONLINE LEARNING NATIONAL COLLEGE OF IRELAND, 2008.

T. Hurley and S. Weibelzahl, 2007. Using MotSaRT to Support On-Line Teachers in Student Motivation. IN: E. Duval, R. Klamma And M. Wolpers, edS., 2ND EUROPEAN CONFERENCE ON TECHNOLOGY ENHANCED EDUCATION (EC-TEL 2007) SPRINGER, 101-111.

J.M. KELLER, 1987. STRATEGIES FOR STIMULATING THE MOTIVATION TO LEARN. PERFORMANCE AND INSTRUCTION, 26 (8), 1-7.

J. Masthoff, 2002. Design AND EVALUATION OF A NAVIGATION AGENT With A MiXED LOCUS OF CONTROL. IN: S.A. CERRI, G. GOUARDÈRES AND F. PARAGUAÇU, EDS., 6TH INTERNATIONAL CONFERENCE ON INTELLIGENT TUTORING SYSTEMS (ITS'2002) LECTURE NOTES IN COMPUTER SCIENCE 2363, SPRINGER-VERLAG, BiARritZ, FrANCE, 982-991.

T. Naps, G. Rößling, J. Anderson, S. CoOper, W. DAnn, R. Fleischer, B. Koldehofe, A. Korhonen, M. Kuittinen, C. Leska, M. McNally, L. Malmi, J. RantakokKo and R.J. Ross, 2003. EVALUATING THE EDUCATIONAL IMPACT OF VISUALIZATION. ACM SIGCSE BULLETIN, 35 (4), 124-136.

K.A. Papanikolaou, M. Grigoriadou, H. Kornilakis and G.D. Magoulas, 2003. PERSONALISING THE INTERACTION IN A Web-BASEd EdUCATIONAL HyPERMEDia SySTEM: THE CASE OF INSPIRE. USER MODELING AND USER ADAPTED INTERACTION, 13 (3), 213-267.

S. Sosnovsky, P. Brusilovsky AND M. Yudelson, 2004. Supporting Adaptive HYPERMEDIA AutHORS With AUTOMATED CONTENT INDEXING. SECOND INTERNATIONAL WORKSHOP ON AUTHORING OF ADAPTIVE AND ADAPTABLE EDUCATIONAL HYPERMEDIA AT THE THIRD INTERNATIONAL CONFERENCE ON ADAPTIVE HYPERMEDIA AND ADAPTIVE WEBBASED Systems (AH'2004), EINDHOVEN, THE NETHERLANDS.

M. SPECHT, 1998. EMPIRICAL EVALUATION OF ADAPTIVE ANNOTATION IN HYPERMEDIA. IN: T. OTTMANN AND I. TOMEK, EDS., ED-MEDIA/ED-TELECOM'98 - 10TH WORLD CONFERENCE on EduCATIONAl Multimedia AND HyPERMEdia AND World CONFERENCE ON Educational Telecommunications AACE, Freiburg, Germany, 1327-1332.

G. WEBER AND P. BRUSILOVSKY, 2001. ELM-ART: AN ADAPTIVE VERSATILE SYSTEM FOR WEB-BASED INSTRUCTION. INTERNATIONAL JOURNAL OF ARTIFICIAL INTELLIGENCE IN EDUCATION, 12 (4), 351-384. 
G. WEBER AND M. SPECHT, 1997. USER MODELING AND ADAPTIVE NAVIGATION SUPPORT IN WWW-BASED TUTORING SYSTEMS. IN: A. JAMESON, C. PARIS AND C. TASSO, EDS., 6TH

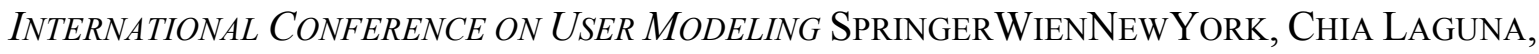
SARDINIA, ITALY, 289-300.

M. Yudelson And P. Brusilovsky, 2005. NAvEx: Providing NAVIGATION SupPort For Adaptive Browsing of Annotated Code Examples. IN: C.-K. Looi, G. McCalla, B. BREDEWEG AND J. BREUKER, EDS., 12TH INTERNATIONAL CONFERENCE ON ARTIFICIAL INTELLIGENCE IN EDUCATION, AI-ED'2005 IOS PRESS, AMSTERDAM, THE NETHERLANDS, 710717.

M. Yudelson, N. Goreva, B.A. MARShall AND D. Goings, 2008. Discovering EDUCATIONAL VALUE OF INTERACTIVE ANNOTATED EXAMPLES IN A BUSINESS PROGRAMMING COURSE. PROCEEDINGS OF THE 48TH ANNUAL IACIS INTERNATIONAL CONFERENCE, SAVANNAH, GEORGIA, USA. 